No letter should be more than 400 words.

For letters on scientific subjects we normally reserve our correspondence columns for those relating to issues discussed recently (within six weeks) in the BMF.

We do not routinely acknowledge letters. Please send a stamped addressed envelope if you would like an acknowledgment.

Because we receive many more letters than we can publish we may shorten those we do print, particularly when we receive several on the same subject.

\section{General practitioners' awareness of COSHH regulations}

SIR, - The Control of Substances Hazardous to Health (COSHH) Regulations became law on 1 October $1989^{\prime}$ and require every employer to assess the risk to health from all chemical and microbiological substances to which their employees are exposed through their work. Appropriate protective measures, including health supervision, must be instituted. The regulations apply equally to self employed people. All people affected by the work-including patients and members of the public - must be considered, although exposure of patients to their own medications is not included.

In practical terms, COSHH regulations require that an inventory should be compiled of all hazardous substances used. An assessment must then be made of the risks associated with these substances. Prevention, control, and monitoring of these risks can then be implemented.

We undertook a survey to establish general practitioners' awareness of the regulations and their compliance with them. We posted a questionnaire to a random selection of 53 general practices; 29 of the questionnaires (54\%) were returned.

Seventeen of the general practitioners had heard of the $\mathrm{COSHH}$ regulations. Eight were aware that they applied to their practice, 10 thought that they did not apply to their practice, and 11 did not know. All the practices but one did not have or were not aware of hazard datasheets. Six practices had informed their staff of hazardous substances, and seven used personal protective equipment. Only one practice kept health records as required by the COSHH regulations.

\section{Health of the nation}

SIR,--J M Harrington's comments on occupational health are trenchant, and many of his proposals are to be welcomed. ${ }^{1}$ There are, however, indications that the United Kingdom is going backwards and not forwards regarding occupational health. Only a few academic departments of occupational medicine and of health and safety have ever existed, and these have recently been further reduced, "rationalised," and closed. There is also serious underfunding and understaffing of field inspectors of the Health and Safety Inspectorate and specialist support. This has paralleled a rapid growth of small and medium sized enterprises in our economy, in which occupational health staff and resources seem unlikely to be a major concern in these difficult financial times.

In the 1990s, despite the activity of a few commercial services and consultancies, there is still woefully inadequate provision of occupational health services in all but our largest companies and
The survey yielded a poor response rate, but there is no reason to believe that the results are not representative of general practices as a whole. It is disturbing that three quarters of the practices surveyed were unaware of the regulations or thought, wrongly, that they did not apply to them. Even more worrying is the finding that no practices had prepared an inventory of hazardous substances. Hence even those that recognised the application of the COSHH regulations did not comply with the first requirement.

Lack of awareness of regulations is no defence in law. All employers, including general practitioners, should be complying with the COSHH regulations. Guidance is available in several documents ${ }^{1 \cdot 3}$ and from the Health and Safety Executive, district occupational health departments, and many independent occupational health and hygiene organisations.

R A COOKE

$S$ GAVAGHAN

Department of Occupational Health,

Worcester Royal Infirmary,

Worcester WRI 3AS

E B HODGSON

University of Oxford

Oxford

Empingham,

Leicestershire LE158PR

1 Health and Safety Commission. The Control of Substances Hazardous to Health Regulations 1988. Approved code of Hazardous to Health Regula

practice. London: HMSO, 1988.
Health and Safety Executive. COSHH assessments. London: HMSO, 1988

3 Glass DC, Hall AJ, Harrington JM. The control of substances hazardous to health: guidance for the initial assessment in hospitals. London: HMSO, 1989.

workplaces. At a time when markets are under close scrutiny elsewhere in the NHS and data on how they work over any length of time are lacking it is pertinent to point out the well documented failure of the marketplace to provide even basic occupational health services for many employees. Perhaps we are going back towards the sweated trade and workshop conditions of Victorian times or "on" to the South East Asian sweatshop model with all that that entails for health and safety in the workplace.

In 1941 the BMA called for "an extension of industrial medical services on both humanitarian and economic grounds" and "for more emphasis on the preventive aspects of industrial medical practice." In 1991 it is surely time that the government implemented the International Labour Organisation's convention on occupational health and committed itself "to developing progressively occupational health services for all workers . . . in all branches of economic activity and all undertakings." "The establishment of a comprehensive national occupational health service, so long advocated by some occupational physicians, is required. Such a service must involve employees (as in Italy) as well as employers and health and safety professionals in its running. Only when employees are directly involved in looking after their own health and working environment will we avoid the deficiencies identified, for instance, in past NHS provision of occupational health services

Workplace Health, Safety, and ANDREW WATTERSON

Environment Research Group

Department of Adult Education,

University of Southampton,

Southampton SO9 5NH

1 Harrington JM. Work related disease and injuries. $B M$ 1991;303:908-10. (12 October.)

2 The new era in industrial medicine [editorial]. BMF 1943; $164-5$.

3 Harrington JM, Gill FS. Occupational health. 2nd ed. Oxford: Blackwell Scientific, 1987:20.

4 Bagnara S, Misiti R, Wintersherger $\mathrm{H}$, eds. Work and health in the 1980s. Berlin: Sigma, 1985.

\section{Seafarers and HIV infection}

SIR, - In his news article on reforming prisons Dr Richard Smith states that the government has promised to review policies for managing prisoners infected with HIV.' Another important group at risk, though often forgotten, are seafarers. Little has been reported regarding the knowledge that seafarers have about HIV infection and AIDS and their behaviour in relation to it.

An inquiry in New York found that 80 seamen had called in at a total of 1124 ports in 45 countries and had intercourse with 615 women. ${ }^{2}$ We recently concluded a retrospective survey of 895 seamen visiting the Seamen's Dispensary at Liverpool during 1985-90; a constant feature noted was heterosexual contact between Polish sailors and contacts in Madagascar in 1986, 1987, 1988, 1989 representing a long term pool of infection (J Dhar et al, meeting of Medical Society for the Study of Venereal Diseases, June 1991, Heidelberg).

Certain characteristics of seafaring result in seafarers being often at high risk. They spend long periods away from home, which provides opportunities for casual sexual behaviour, often in areas where there is a known high prevalence of HIV and condoms are frequently not available or reliable; prostitution being an accepted feature of port life may be another factor. If seafarers require medical attention in some countries they are undoubtedly at increased risk of contracting HIV infection from needles and syringes, etc.

In Valencia in 1987 a study found that of 290 seafarers tested for HIV, seven were seropositive; five of these were drug users and two had contracted the infection through heterosexual contact. This prevalence is much higher than that in the general population. Van Damme and Van Damme under- 